\title{
Structural Verification and Modeling of a Tension Cone Inflatable Aerodynamic Decelerator
}

\author{
Christopher L. Tanner ${ }^{*}$ \\ Georgia Institute of Technology, Atlanta, GA 30332-0150 \\ Juan R. Cruz ${ }^{\dagger}$ \\ NASA Langley Research Center, Hampton, VA 23681-2199 \\ and \\ Robert D. Braun \\ Georgia Institute of Technology, Atlanta, GA 30332-0150
}

\begin{abstract}
Verification analyses were conducted on membrane structures pertaining to a tension cone inflatable aerodynamic decelerator using the analysis code LS-DYNA. The responses of three structures - a cylinder, torus, and tension shell - were compared against linear theory for various loading cases. Stress distribution, buckling behavior, and wrinkling behavior were investigated. In general, agreement between theory and LS-DYNA was very good for all cases investigated. These verification cases exposed the important effects of using a linear elastic liner in membrane structures under compression. Finally, a tension cone wind tunnel test article is modeled in LS-DYNA for which preliminary results are presented. Unlike data from supersonic wind tunnel testing, the segmented tension shell and torus experienced oscillatory behavior when subjected to a steady aerodynamic pressure distribution. This work is presented as a work in progress towards development of a fluid-structures interaction mechanism to investigate aeroelastic behavior of inflatable aerodynamic decelerators.
\end{abstract}

\section{Nomenclature}

$\begin{array}{ll}b & =\text { arc length of wrinkled region } \\ B^{2} & =\text { tension shell shape factor } \\ c & =\text { torus radius ratio }\left(r_{m} / r_{M}\right) \\ C_{p} & =\text { pressure coefficient } \\ E & =\text { elastic modulus } \\ F_{c r} & =\text { critical buckling load } \\ G & =\text { shear modulus } \\ l & =\text { length } \\ M & =\text { applied moment } \\ p & =\text { internal pressure } \\ p_{a} & =\text { aerodynamic pressure distribution } \\ p_{0} & =\text { uniform pressure distribution } \\ P & =\text { force due to internal pressure }\left(p \pi r^{2}\right) \\ r & =\text { radius or tension shell radial coordinate } \\ r_{b} & =\text { tension shell base radius } \\ r_{m} & =\text { minor torus radius }\end{array}$

\footnotetext{
* Graduate Research Assistant, Daniel Guggenheim School of Aerospace Engineering, AIAA Student Member, christopher.tanner@gatech.edu

${ }^{\dagger}$ Aerospace Engineer, Atmospheric Flight and Entry Systems Branch, AIAA Member, Juan.R.Cruz@nasa.gov

* Associate Professor, Daniel Guggenheim School of Aerospace Engineering, AIAA Fellow, robert.braun@ae.gatech.edu
} 


$\begin{array}{ll}r_{M} & =\text { major torus radius } \\ t & =\text { material thickness } \\ x, y, z & =\text { Cartesian coordinates for cylinder } \\ z & =\text { tension shell axial coordinate } \\ \alpha & =\text { tension shell stress ratio }\left(\sigma_{c} / \sigma_{m}\right) \\ \varepsilon_{c} & =\text { circumferential strain } \\ \kappa & =\text { cylinder curvature due to load } \\ \phi & =\text { angle measurement around torus cross-section } \\ v & =\text { Poisson's ratio } \\ \sigma_{0} & =\text { stress at base of tension shell } \\ \sigma_{c} & =\text { circumferential stress } \\ \sigma_{l} & =\text { longitudinal stress } \\ \sigma_{m} & =\text { meridional stress } \\ \text { FSI } & =\text { Fluid-Structures Interaction } \\ \text { IAD } & =\text { Inflatable Aerodynamic Decelerator } \\ \text { LSTC } & =\text { Livermore Software Technology Corporation }\end{array}$

\section{Introduction}

nflatable aerodynamic decelerator (IAD) designs, such as the tension cone shown in Figure 1, will require fluid-structure interaction (FSI) analyses to appropriately assess structural response at relevant aerodynamic conditions and scales. However, given the complexity of FSI, it is often useful to analyze portions of the problem under simplified conditions rather than immediately attempting to analyze the full problem. This process can lead to a better understanding of the underlying physics and the behavior of the computational tools used to model those physics.

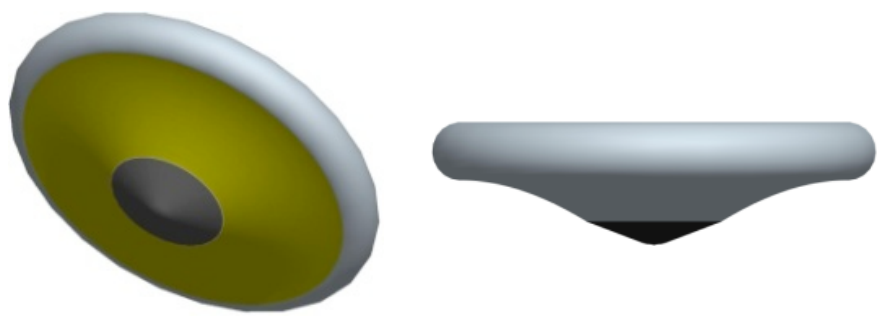

Figure 1. Tension cone IAD (figure from Ref. 1).

A tension cone IAD consists of a shell of revolution, referred to as a tension shell attached at one end to the forebody of a blunt entry vehicle, and at the other end to a pressurized, toroidal structural member. The tension shell is intentionally shaped to carry only tensile loads in the meridional and circumferential directions while transferring compressive loads to the torus. Inflatable aerodynamic decelerators such as the tension cone are meant for supersonic deceleration, particularly in thin atmospheres, and will likely be constructed from high-strength textile materials with impermeable liners or coatings.

This document summarizes a verification of the analysis code LS-DYNA ${ }^{2}$, developed by the Livermore Software Technology Corporation (LSTC), as applied to structures relevant to tension cone IADs. The tension cone is reduced to simple geometries -- a cylinder, torus, and tension shell -- which have well-understood static behavior. Analytical relationships that predict stress distributions, buckling loads, and wrinkling behavior for these shapes are used as a benchmark for comparison with LS-DYNA. These simple cases bring out the effects of particular modeling parameters in LS-DYNA, with various geometries and loading scenarios exercising different aspects of the code. Following verification, a preliminary structural analysis is performed on a tension cone IAD that is representative of a fully inflatable tension cone wind tunnel model tested in the 10- by 10 - $\mathrm{ft}$ Supersonic Wind Tunnel at the NASA 
Glenn Research Center. ${ }^{1}$ This work represents the initial structural verification of LS-DYNA, as part of a larger FSI effort intended to predict the aeroelastic behavior of IADs in supersonic flowfields.

\section{Textile Modeling Parameters}

Unless otherwise stated, all of the following verification cases were performed using the same material and finite element parameters stated here to maintain consistency across all solutions. The textile portion of each structure was modeled using membrane shell elements 0.01315 inches thick. The mat_fabric material model was used assuming the following isotropic properties: elastic modulus $(E)$ of $387.7 \mathrm{ksi}$, Poisson's ratio $(v)$ of 0.3 , and shear modulus $(G)$ determined from $G=0.5 E /(1+v)$. The fabric material model invokes an alternate membrane element algorithm using one through-thickness integration point regardless of what is specified in the section_shell keyword.

LSTC recommends adding a "liner" to help stabilize membrane elements that have gone into compression. The liner is a linear elastic material that augment the element's original material definition, effectively increasing tensile stiffness and making compressive stiffness non-zero, but maintaining zero bending stiffness in the element. Since the verification cases involve matching analytic theories (which assume zero compressive stiffness) and are pseudo-static, a liner was omitted from the models. LSTC also recommends adding at least 5\% Rayleigh damping (corresponding to $5 \%$ of the critical damping value) to increase the stability of membrane elements. However, loaded elements still appeared to experience oscillatory behavior with low damping. Thus, in the attempt to obtain pseudo-static results, damping was increased to $100 \%$ of critical damping. The LS-DYNA keyword parameters described above are presented in card format in Table 1.

Table 1. LS-DYNA keyword parameters for fabric portion of all verification cases.

\begin{tabular}{|c|c|c|c|c|c|c|c|c|c|}
\hline \multicolumn{10}{|c|}{ *SECTION_SHELL } \\
\hline secid & elform & \multicolumn{2}{|r|}{ shrf } & nip & propt & \multicolumn{2}{|c|}{ qr/irid } & i comp & setyp \\
\hline 1 & 5 & & 1 & 1 & & 1 & 0 & 0 & 1 \\
\hline t1 & t2 & & t3 & t4 & $\mathrm{nl}$ & & marea & idof & edgset \\
\hline 0.01315 & 0.01315 & 0. & 1315 & 0.01315 & & 0 & 0 & 0 & 0 \\
\hline *MAT & _FABRIC & & & & & & & & \\
\hline mid & & ro & ea & $e b$ & ec & prba & prca & & $\mathrm{rcb}$ \\
\hline 1 & $1.0544 \mathrm{E}$ & -4 & 387705 & 0 & 0 & 0.3 & 0 & $\partial$ & 0 \\
\hline$g a b$ & & $\mathrm{bc}$ & gca & cse & el & prl & lratio & & amp \\
\hline 0 & & 0 & 0 & 1 & 0 & 0 & 0 & $\partial$ & 1.0 \\
\hline aopt & & lc & fac & ela & $\ln r c$ & form & fvopt & tsr & fac \\
\hline 0 & & 0 & 0 & 0 & 0 & 0 & 0 & 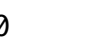 & 0 \\
\hline$x p$ & & yp & $z p$ & $a 1$ & $a 2$ & a3 & $x d$ & & $x l$ \\
\hline 0 & & 0 & 0 & 0 & 0 & 0 & 0 & $\partial$ & 0 \\
\hline
\end{tabular}

All cases were solved explicitly using the double precision, symmetric multiprocessor version of the LS-DYNA v971, release 4.2. Generally explicit codes are intended for dynamic problems that have time dependence and are not generally used for pseudo-static problems. LS-DYNA does have an implicit solving routine that is more suited for static problems. However, membrane elements are not implemented in LS-DYNA's implicit routine, which immediately precludes its use for this study. To effectively use an explicit solver for the following pseudo-static cases: (a) loads must be applied smoothly, (b) energy balance must be monitored to ensure that the systems energy is due almost entirely to internal energy, not kinetic energy, and (c) damping must be used with care to create a static solution in a short period of time without significantly influencing the end result.

\section{Column Buckling and Membrane Wrinkling}

Two circular cylinders were constructed to perform buckling and wrinkling verification of LS-DYNA. For a tension cone IAD, buckling prediction is particularly important as it is a critical collapse mode which drives the required torus internal pressure and consequently its mass. For the same reasons, wrinkling is an important response. Cylinder geometry and nomenclature are given in Figure 2. The Cartesian coordinate system originates from the base of the cylinder along the axis of symmetry. The radius for all cylinders is 1.5 inches. 


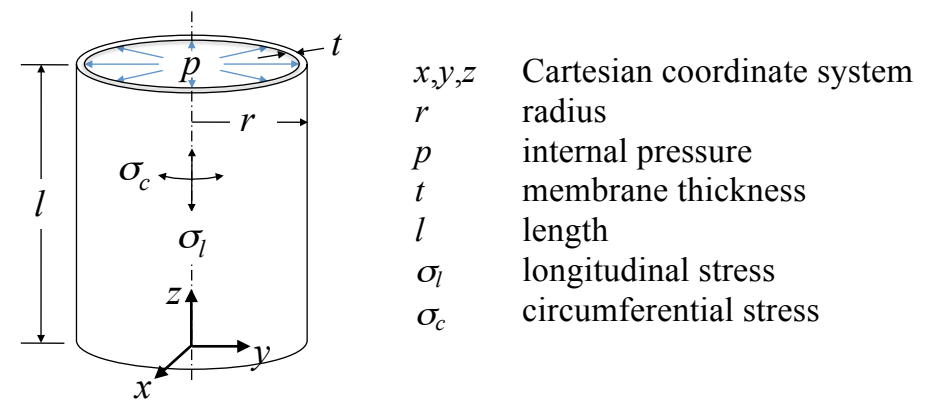

Figure 2. Cylinder geometry and nomenclature.

\section{A. Column Buckling}

A column 45 inches in length was modeled to investigate buckling behavior. Column end caps were modeled as stiff plates. The bottom cap was constrained in translation but permitted to rotate only about the $x$-axis; the top cap was only permitted to translate along the $z$-axis and rotate about the $x$-axis. An internal pressure of 200 psi was imposed to achieve a configuration that exhibited global buckling while retaining tensile longitudinal stress when buckled. The radius of the un-inflated shape was reduced to ensure that the final, inflated radius was close to 1.5 inches. Fichter ${ }^{3}$ approximated the critical buckling load of a column with membrane walls subject to internal pressure assuming the cylinder walls remain in tension and the column's cross-section remains circular throughout. Fichter's relationship for the critical buckling load is

$$
F_{c r}=\frac{\left(E I \pi^{2} l^{-2}\right)\left(p \pi r^{2}+G \pi r t\right)}{E I \pi^{2} l^{-2}+p \pi r^{2}+G \pi r t}
$$

One issue common to buckling analysis using finite element analysis is that imperfection needs to be introduced into the model in order to recover an appropriate buckling load. Thus, for this model, imperfection was introduced as an applied moment couple at both ends of the column at various magnitudes. Buckling results are given in Figure 3. Figure $3 \mathrm{~b}$ shows that the buckling load with no end moment overshoots Fichter's value and experiences substantial oscillation after buckling has occurred. The addition of small moments at the ends of the columns decrease the buckling load overshoot and subsequent oscillations. Oscillation is due to the release of potential energy after buckling. A $10 \mathrm{~Hz}$ Butterworth filter was applied to results to extract a pseudo-static buckling load as shown in Figure $3 \mathrm{~b}$. Even though the peak buckling load varies considerably with the applied moment, the steady-state buckled load appears to be within about $4 \%$ of that predicted by Fichter's theory for all cases.

\section{B. Membrane Wrinkling - Cylinder Compresion}

A cylinder six inches in length was modeled to investigate its wrinkling response (i.e., zero wall stress condition). The column was subjected to $20 \mathrm{psi}$ of internal pressure. Boundary conditions were a fixed lower end cap, and an upper end cap that was only allowed to translate axially. In Figure 4 the end cap externally applied force versus stroke response is plotted. The black line represents axial force due to internal pressure, where internal pressure is linearly dependent on the cylinder's volume, which varies as stroke increases. For a cylinder with membrane walls, the externally applied force should be equal to the internal force after the walls have wrinkled. Indeed, LS-DYNA recovers this force when no liner is present after the cylinder walls have wrinkled. Wall oscillation occurs just after wrinkling and ceases when the cylinder compresses into a stable configuration with walls deflected outward, as in Figure 5c. 

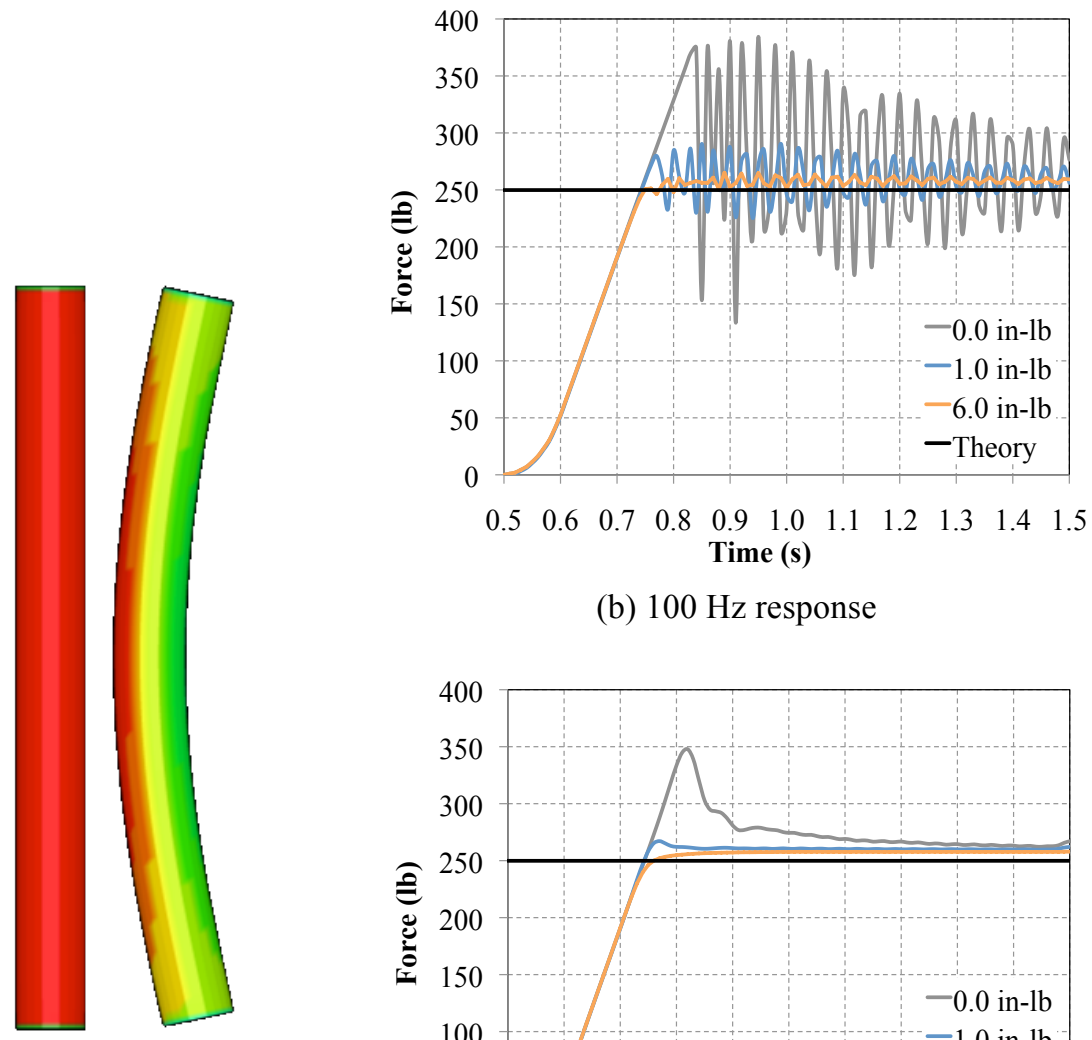

(b) $100 \mathrm{~Hz}$ response

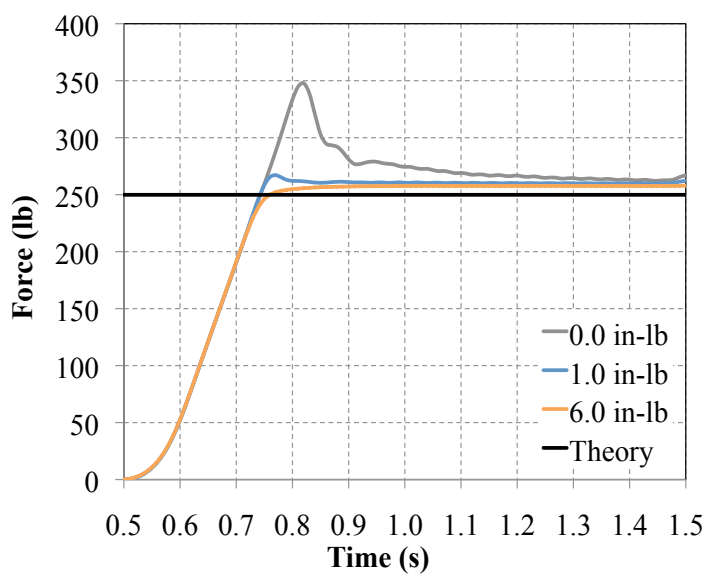

(a) Contours of $\sigma_{1}$

(c) Filtered response

Figure 3. Column buckling response for various moment couples.

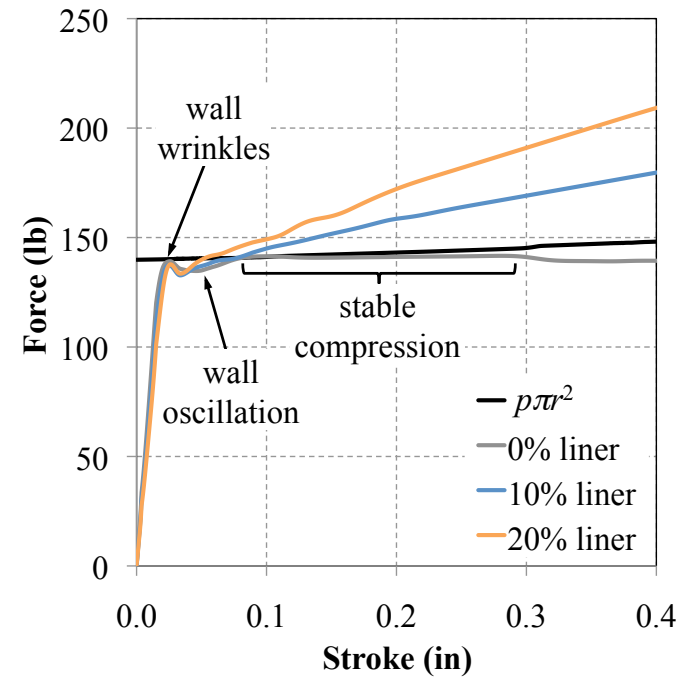

Figure 4. Structural response of a short cylinder using for various liner thicknesses. 


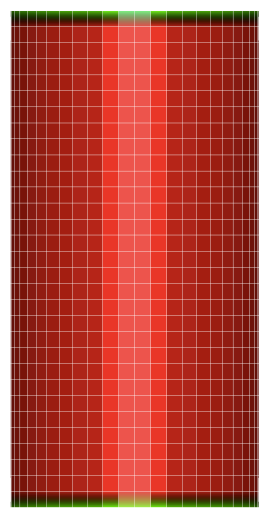

(a) Inflated, pre-loaded

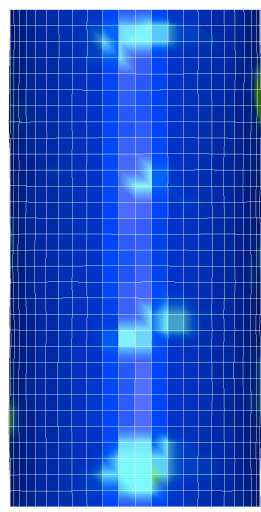

(b) Wall oscillation

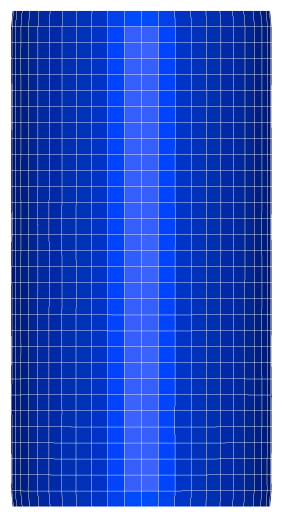

(c) Stable compression

Figure 5. Cylinder buckling progression.

The addition of a linear elastic liner significantly increases the amount of external force required for a given stroke after the cylinder walls have wrinkled. Reaction force is a function of both the internal pressure as well as the compressive load carried by the cylinder walls. Greater external force is required during compression since the liner, despite its small elastic modulus and thickness with respect to the base material, has compressive strength and will carry a non-negligible amount of load. The liner is defined by an elastic modulus $10 \%$ of the base material $\left(E_{\text {liner }}=0.10 E\right)$ and a Poisson's ratio equal to that of the base material. Liner thickness is a fraction of the base material's thickness (i.e., a $10 \%$ liner corresponds to $t_{\text {liner }}=0.10 t$ ).

\section{Membrane Wrinkling - Cylinder Bending}

A cylinder six inches in length was subjected to 30 psi of internal pressure and a linearly increasing moment couple. The ends, modeled as rigid shells, were assigned boundary conditions similar to the column in Section II.A. Stein and Hedgpeth ${ }^{4}$ developed a relationship that relates an applied moment couple to the curvature of the column, effectively showing that a pressurized cylinder is able to maintain a stable, static configuration in a partially wrinkled state. This is an important result for the tension cone as it implies that a partially wrinkled configuration will continue to support load. Figure 6 provides additional nomenclature and compares results against Stein and Hedgepeth's theory. In Figure 6a, the parameter $b$ is an arc length, measured from the bottom of the cylinder at $b=0$, to the edge of the wrinkled region, with a maximum length of $b=\pi r$; in other words, it is half of the total arc length of the wrinkled region.

For the material established in Section II, LS-DYNA results almost exactly match theory. This test case also helps illustrate the liner explored in Section III.B. Figure 6c indicates that wrinkling response is approximately the same for all cases until $b / \pi r=1 / 2$. The liner then begins to have a noticeable effect, requiring larger moments for a given curvature, despite its low modulus and thickness relative to the base material. These results illustrate that liner effects may be non-negligible when a structure is in a wrinkled condition. This corroborates the results in Section II.B and reinforces that implementing a liner must be done with care since the structural response may deviate from the actual response.

\footnotetext{
${ }^{*}$ In Reference 4 a photograph is shown of a cylinder in just such a partially wrinkled configuration, showing that this is a physically possible state.
} 


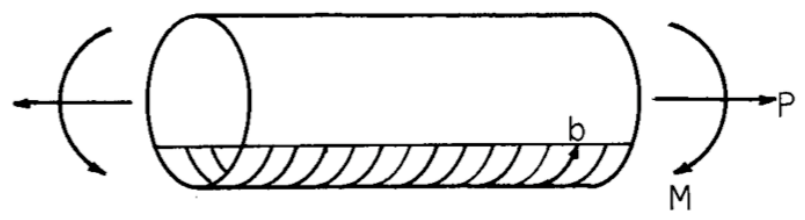

(a) Column post-buckling nomenclature (figure from Ref. 4)
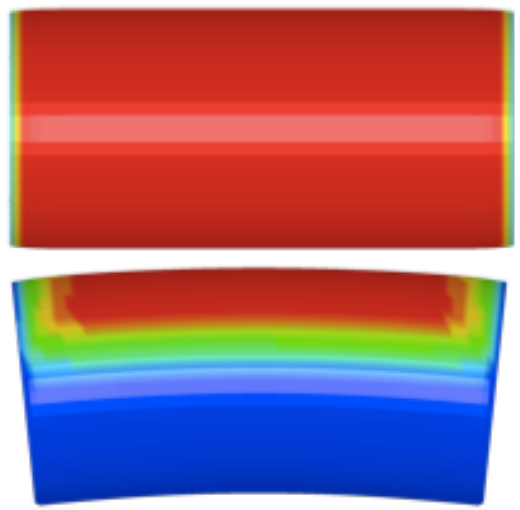

(b) Contours of $\sigma_{l}$

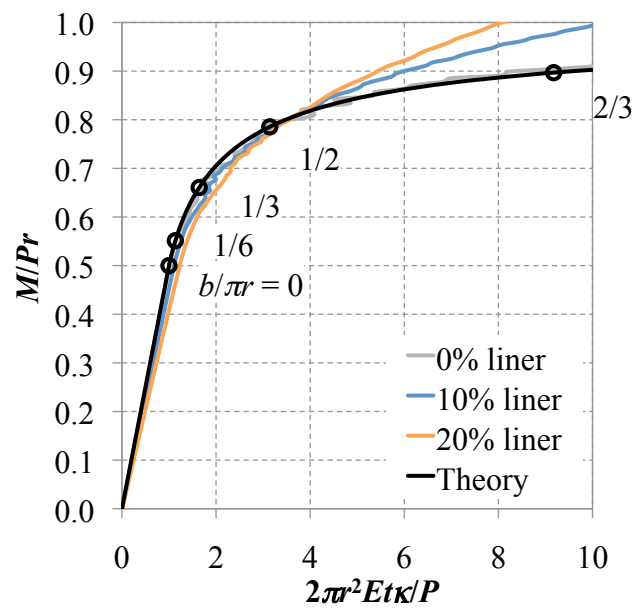

(c) Moment-curvature response to various liner thicknesses

Figure 6. Column post-buckling results for various liner thicknesses.

\section{Torus Stress Distribution}

For the tension cone IAD, a pressurized torus serves as a primary load-bearing member, reacting the compressive loads transmitted through the tension shell. Accurate prediction of the stress distribution of a torus translates directly into material requirements and mass estimation of the tension cone. ${ }^{5}$ Torus geometry and nomenclature is provided in Figure 7.

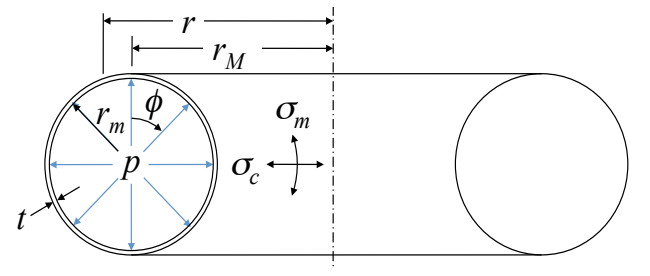

$\begin{array}{ll}r_{M} & \text { major radius } \\ r_{m} & \text { minor radius } \\ p & \text { internal pressure } \\ t & \text { thickness } \\ \phi & \text { angle around cross-section } \\ \sigma_{m} & \text { meridional stress } \\ \sigma_{c} & \text { circumferential stress }\end{array}$

Figure 7. Torus geometry and nomenclature.

Linear theory for the stress distribution in a toroidal shell relies on the assumption that the major torus radius is much larger than the minor torus radius. ${ }^{6}$ In this scenario, a section of the torus appears as a cylinder and the circumferential stress can be approximated as a constant value throughout the shell. For cases in which the torus radius ratio $\left(c=r_{m} / r_{M}\right)$ is not small, circumferential stress becomes nonlinear. Sanders ${ }^{7}$ presents a nonlinear theory for accurately predicting torus stresses in both the meridional and circumferential directions. 


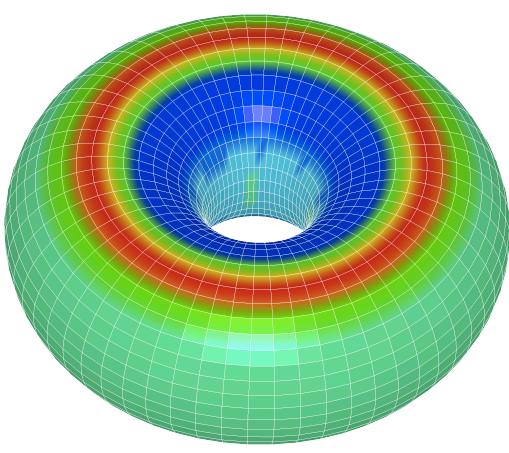

(a) Contours of $\sigma_{c}$

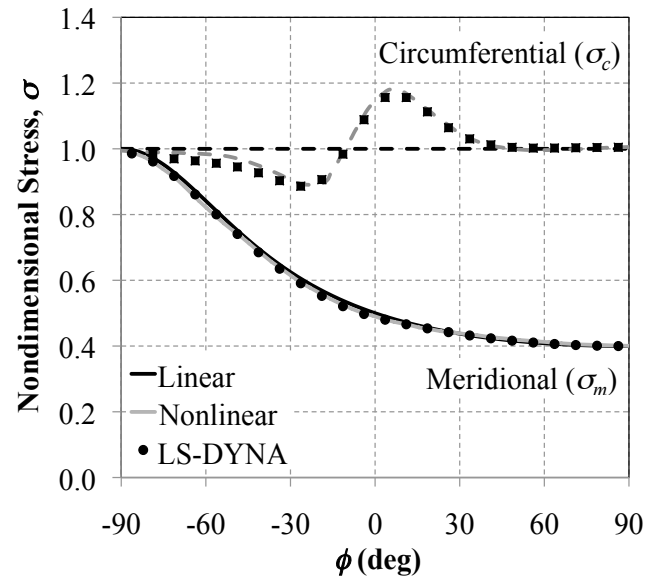

(b) Normalized torus stress distribution

Figure 8. Comparison of linear, nonlinear, and computational stress for $c=2 / 3$. Stress is non-dimensionalized by the maximum linear stress in each direction.

In Figure 8 solid lines represents analytical predictions in the meridional direction, dotted lines represents analytical predictions in the circumferential direction, with black being used for the linear theory and grey being used for the nonlinear theory. Discrete symbols represent LS-DYNA solutions. Figure 8 shows that finite element results are nearly identical to the nonlinear solution for both the circumferential and meridional stresses. However, linear theory in the circumferential direction predicts the incorrect behavior and differs from the nonlinear and computational results by up to $18 \%$.

\section{Tension Shell Stress Distribution}

The tension shell as discussed here is a conical structure as proposed by Anderson et. al. ${ }^{8}$ for mass efficient decelerator concepts. The tension shell shape, given in Figure 9, can be obtained assuming a constant stress ratio $\left(\alpha=\sigma_{c} / \sigma_{m}\right)$, a shape parameter, and an axisymmetric aerodynamic pressure distribution $\left(p_{a}\right)$.

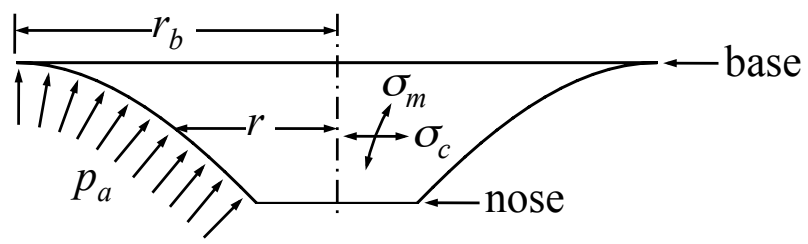

Figure 9. Tension shell geometry and nomenclature.

\section{A. Boundary Conditions}

In modeling the tension shell for this verification study the nose and base perimeters of the tension shell are constrained from moving in the radial direction. A radial constraint at the nose and base implies that the circumferential strain, $\varepsilon_{c}$, is zero at these edges. For the isotropic material used in this verification study

$$
\varepsilon_{c}=\frac{\sigma_{c}-v \sigma_{m}}{E}
$$

However, since $\varepsilon_{c}=0$ at the boundaries of the tension shell, Equation (2) yields $\sigma_{c} / \sigma_{m}=v$. The tension shell theory defines a tension shell by a constant stress ratio, $\sigma_{c} / \sigma_{m}=\alpha$. Thus, in order for the verification case and tension shell theory to agree, $\alpha=v$. Per Section II, the material properties used for this study assume $v=0.3$; as such, the tension shell shape used for this study is defined by a stress ratio of 0.3 . In addition to these radial constraints, the nose is fully constrained in axial translation, but the base is allowed to translate axially. 


\section{B. Stress Distribution}

For analytic pressure distributions, such as a uniform or Newtonian distribution, the tension shell profile and subsequent stress state can be derived from the tension shell theory. For a uniform pressure distribution, meridional stress $\left(\sigma_{m}\right)$ is a function of the stress at the tension shell base $\left(\sigma_{0}\right)$ and circumferential stress is a prescribed fraction of the meridional stress $(\alpha)$. These relationships, from reference 8, are given in Equations (3) where $B^{2}$ is the tension shell shape factor and $p_{0}$ is a constant, uniform pressure.

$$
\begin{aligned}
\sigma_{0} & =\frac{p_{0} r_{b}}{2 B^{2} t} \\
\sigma_{m} & =\sigma_{0}\left(\frac{r_{b}}{r}\right)^{1-\alpha} \\
\sigma_{c} & =\alpha \sigma_{m}
\end{aligned}
$$

For these analyses, a uniform mesh was prescribed on a tension shell defined by $\alpha=0.3$ and $B^{2}=0.5$. The tension shell coordinates were obtained using the procedure described in reference 8 .

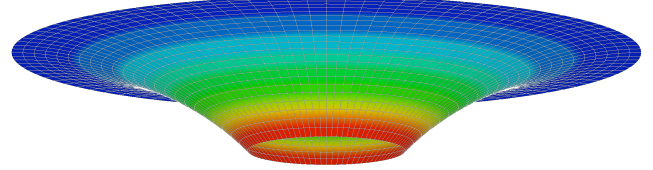

(a) contours of $\sigma_{m}$

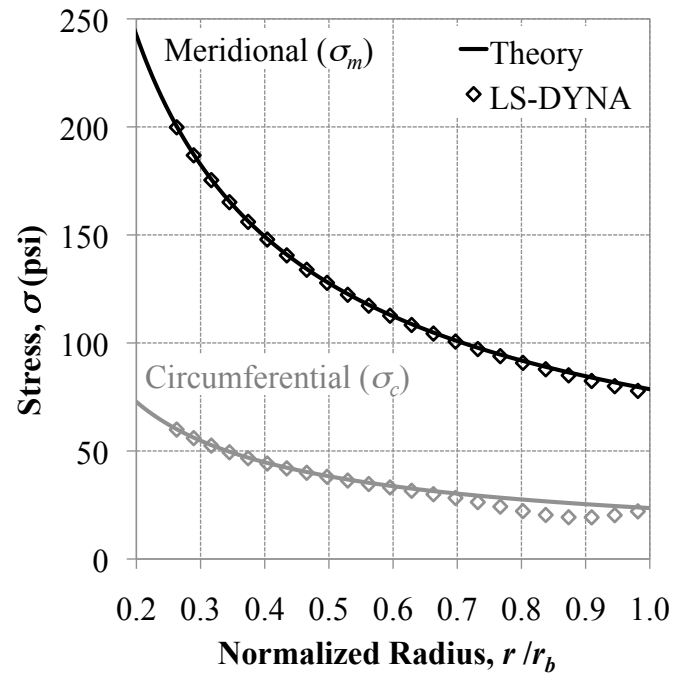

(b) Tension shell stress distribution

Figure 10. Tension shell stress distribution results for $\alpha=0.3, B^{2}=0.5$, and $p_{0}=0.1 \mathrm{psi}$.

Figure 10b shows that the meridional stresses predicted by LS-DYNA are well behaved and follow the linear theory solution almost exactly. The circumferential stresses predicted by LS-DYNA agree with the linear theory solution up to a $r / r_{\mathrm{b}}$ of 0.7. After this point, finite element results deviate from the linear solution. It is likely that this deviation is due to deformation of the tension shell geometry in the LS-DYNA model as compared to the shape specified in the linear theory; quoting from reference 8: "It should be noted that the circumferential stress $N_{\theta}$ is very sensitive to small changes in the shape in the vicinity of the base; this sensitivity may require special consideration in the vehicle design." (p. 12). In general agreement between the linear theory and the nonlinear LS-DYNA results should improve when deformations are negligible, as $p_{0}$ approaches zero and/or in the limit of a very stiff tension shell. In performing the work presented here, the sensitivity of the results to the exact shape of the tension shell was also observed near the nose.

\section{Tension Cone Structural Model}

The tension cone shown in Figure 11 was tested in the 10- by 10-ft Supersonic Wind Tunnel at the NASA Glenn Research Center. ${ }^{1}$ 


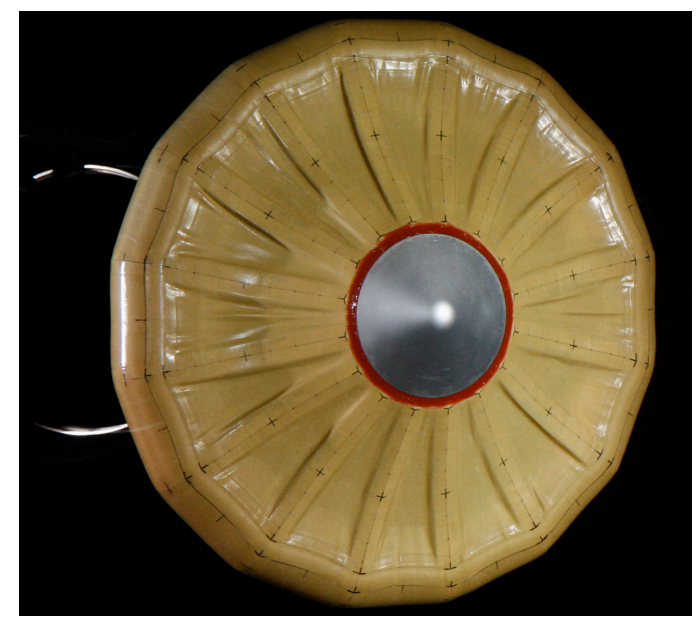

(a) Front view (photo from Ref. 1)

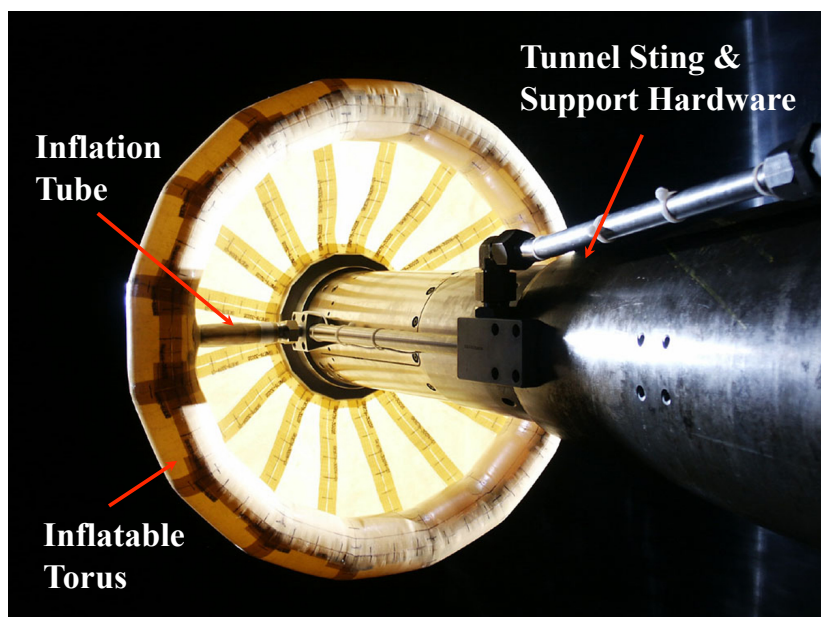

(b) Back view (photo from Ref.5)

Figure 11. Inflatable tension cone model.

A continuous tension cone was approximated by a 16-sided polygon constructed from urethane-coated Kevlar ${ }^{\circledR}$ fabric. The purpose of the urethane coating was to eliminate fabric porosity and permit gore seams to be welded together instead of sewn. The welding process involved butting two seam ends together with a second layer of the material and heating until the urethane bonded all pieces together. To properly seal all seams and ensure structural integrity, the tension cone employed between one and four layers of welded material throughout the model. A detailed finite element model consisting of over 16,000 elements was constructed of this tension cone model. The colored regions in the mesh shown in Figure 12 capture the various material thicknesses. The Kevlar ${ }^{\circledR}$ fabric is modeled identically to the verification cases except that the thickness is increased as appropriate. The inflation tubes and metallic hardware are fully constrained as this represents the mounting configuration in the wind tunnel.

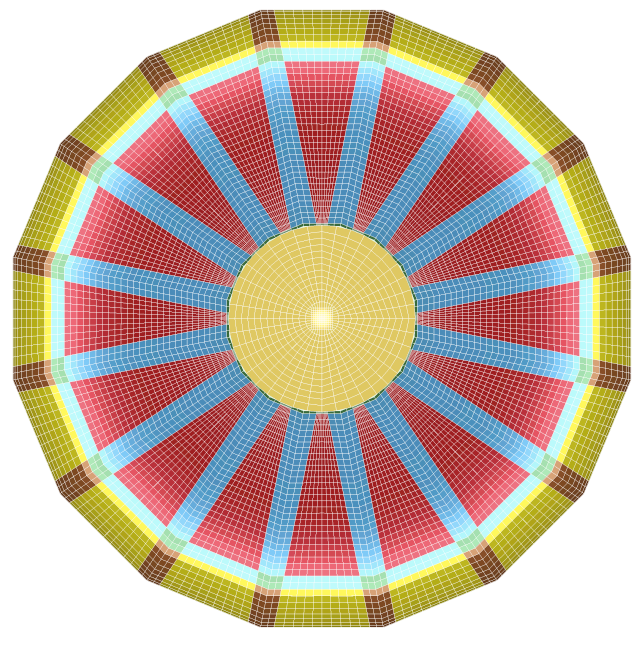

(a) front view

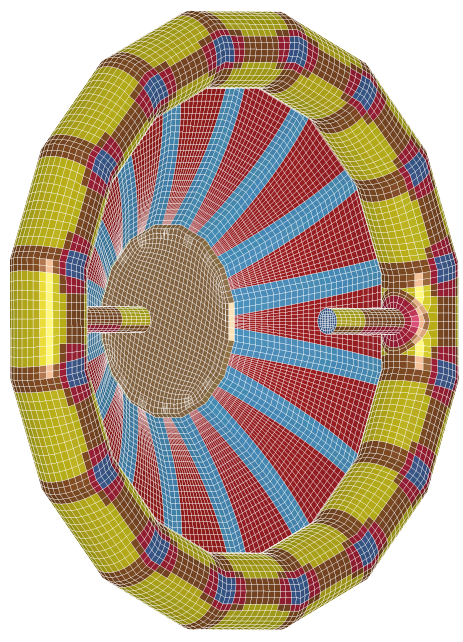

(b) back view

Figure 12. Detailed finite element mesh of tension cone.

Preliminary aeroelastic response of the tension cone was obtained by first pressurizing the torus to 50 psi and then imposing the aerodynamic pressure distribution shown in Figure 13 on the tension shell and torus. The applied pressure distribution was obtained from rigid tension cone testing in the Unitary Plan Wind Tunnel at NASA Langley Research Center. ${ }^{1}$ 


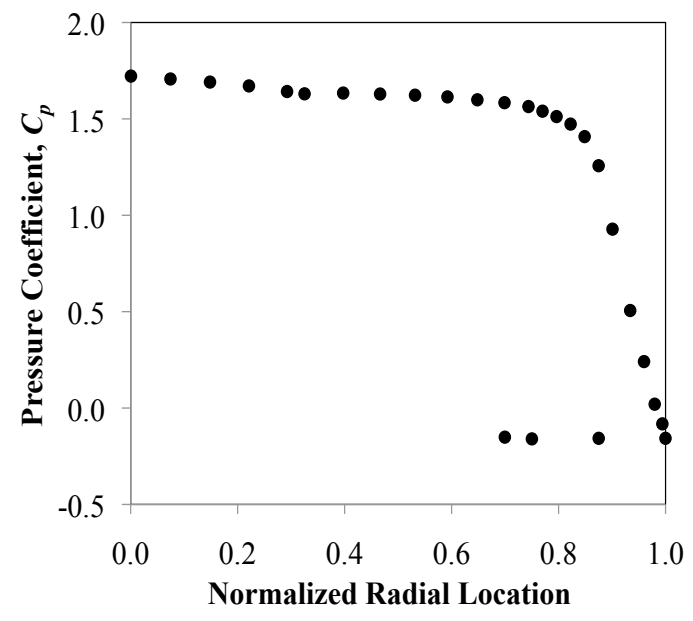

Figure 13. Pressure distribution at Mach 2.5, adapted from Ref 5.

The tension shell vibrated in response to the applied load. Similarly, the torus appeared to flex and oscillate about the axis formed by the inflation tubes. In general, the tension cone exhibited an unsteady response to a steady aerodynamic load, which contrasts the steady behavior observed during wind tunnel testing. The addition of a $10 \%$ liner eliminated torus the motion, but the tension shell vibration remained. A snapshot of the tension cone's deformation is shown in Figure 14 using contours of axial displacement.

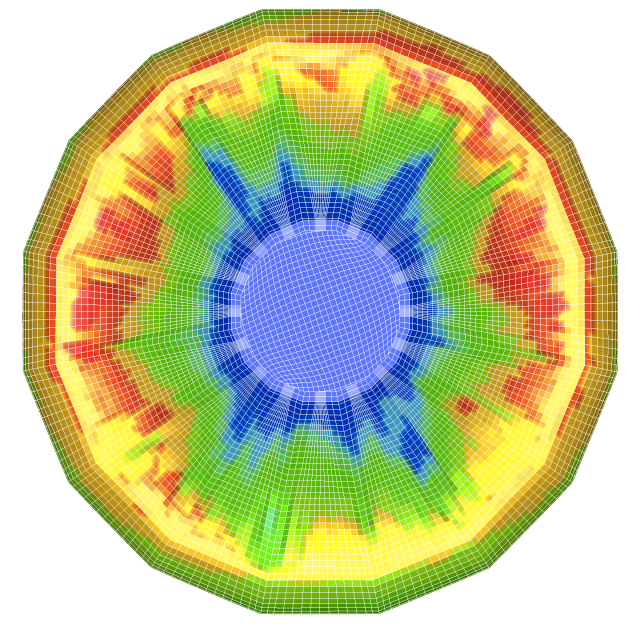

(a) front view

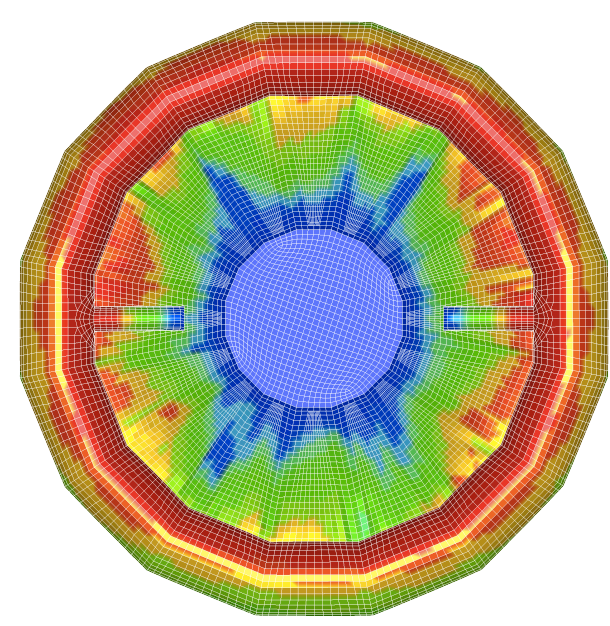

(b) back view

Figure 14. Tension cone contours of axial displacement.

The presented results are very preliminary, as the applied pressure distribution remained constant even as the structure deformed. Damping will be necessary to eliminate the vibrations on the tension shell and recover the static behavior observed during the wind tunnel test. Additionally, the material properties used are those described in Section II, which result in a shear modulus of $150 \mathrm{ksi}$. In contrast, the actual Kevlar ${ }^{\mathbb{B}}$ material is orthotropic and testing indicates that the material may have a shear modulus closer to $5 \mathrm{ksi}^{9}$, over an order of magnitude lower than the value used in this preliminary tension cone analysis.

\section{Summary and Future Work}

Several basic geometries and loading scenarios were simulated using the finite element analysis code LS-DYNA. The analysis code was verified against known analytical theories, both linear and nonlinear, using a single material and a finite element definition that did not utilize a liner. A liner was introduced into the wrinkling analysis to 
illustrate how it augments the structure's stiffness in situations where the membrane is placed in compression. Finally, a 16-segment tension cone wind tunnel test article is modeled with finite element boundaries along seam boundaries. Preliminary analysis was performed on the tension cone using a representative, static aerodynamic pressure distribution. The tension cone exhibited unsteady behavior to a steady load, which contradicts the steady behavior observed during wind tunnel testing. Introduction of damping and more appropriate material properties into the model will be necessary to create a more accurate representation of wind tunnel results.

This paper is presented as a work in progress. To further mature the tension cone structural model, implementation of oriented, orthotropic, nonlinear materials on simple models and validation of those models against experimental results will be required. Following a validation of nonlinear material models, work will begin on implementing the segmented tension cone structural model presented in Section VI in a fluid dynamics solver. Difficulties in this area involve mapping the aerodynamic and structural grids to each other as well as implementing a scheme to control grid stretching or remeshing as the structure deforms. Additionally, it is not known how much of the tension cone's aft structure will need to be modeled to recover accurate pressures. Joint implementation of accurate structural response and accurate aerodynamic pressures, which change with structural deformation, will be required to recover tension cone wind tunnel behavior. The fluid-structure interaction mechanism described above will be used to validate structural response against the static aeroelastic shape of the tension cone at various angles of attack as well as buckling response of the tension cone as torus pressure is reduced.

\section{References}

${ }^{1}$ Clark, I. G., Cruz, J. R., Hughes, M. F., Ware, J. S., Madlangbayan, A., Braun, R. D., “Aerodynamic and Aeroelastic Characteristics of a Tension Cone Inflatable Aerodynamic Decelerator," AIAA Aerodynamic Decelerator Systems Technology Conference and Seminar, AIAA-2009-2967, May 2009.

${ }^{2}$ LS-DYNA Keyword User's Manual, Version 971, Vol. I-II, May 2007.

${ }^{3}$ Fichter, W. B., "A Theory for Inflated Thin-Wall Cylindrical Beams,” NASA TN D-3466, 1966.

${ }^{4}$ Stein, M. and Hedgepeth, J. M., “Analysis of Partly Wrinkled Membranes,” NASA TN D-813, 1961.

${ }^{5}$ Clark, I. G., Aerodynamic Design, Analyses, and Validation of a Supersonic Inflatable Aerodynamic Decelerator, Ph.D. Dissertation, Georgia Institute of Technology, August 2008.

${ }^{6}$ Young, W., C., Roark's Formulas for Stress and Strain, McGraw-Hill Companies, 2002, p. 593.

${ }^{7}$ Sanders, J. L. and Liepins, A., A., "Toroidal Membrane Under Internal Pressure," AIAA Journal, Vol. 1, No. 9, September 1963, pp. 2105-2110.

${ }^{8}$ Anderson, M. S., Robinson J. C., Bush, H. G., and Fralich, R. W., "A Tension Shell Structure for Application to Entry Vehicles," NASA TN D-2875, 1965.

${ }^{9}$ Hutchings, A. L., Braun, R. D., Masuyama, K. "Experimental Determination of Material Properties for Inflatable Aeroshell Structures," AIAA Aerodynamic Decelerator Systems Technology Conference and Seminar, AIAA-2009-2949, May 2009. 